\title{
Interleukin-17A induces glucocorticoid insensitivity in human bronchial epithelial
} cells

\author{
G.J. Zijlstra*, N.H.T. ten Hacken\#, R.F. Hoffmann*, A.J.M. van \\ Oosterhout*, and I.H. Heijink*,\#,
}

ABSTRACT: A subset of asthma patients suffer from glucocorticoid (GC) insensitivity. T-helper cell type 17 cells have an emerging role in GC insensitivity, although the mechanisms are still poorly understood.

We investigated whether interleukin (IL)-17A induces GC insensitivity in airway epithelium by studying its effects on responsiveness of tumour necrosis factor (TNF)- $\alpha$-induced IL-8 production to budesonide in human bronchial epithelial 16HBE cells. We unravelled the underlying mechanism by the use of specific pathway inhibitors, reporter and overexpression constructs and a histone deacetylase (HDAC) activity assay.

We demonstrated that IL-17A-induced IL-8 production is normally sensitive to GCs, while IL-17A pre-treatment significantly reduced the sensitivity of TNF- $\alpha$-induced IL-8 production to budesonide. IL-17A activated the p38, extracellular signal-related kinase (ERK) and phosphoinositide-3-kinase (PI3K) pathways, and the latter appeared to be involved in IL-17A-induced GC insensitivity. Furthermore, IL-17A reduced HDAC activity, and overexpression of HDAC2 reversed IL-17Ainduced GC insensitivity. In contrast, IL-17A did not affect budesonide-induced transcriptional activity of the GC receptor, suggesting that IL-17A does not impair the actions of the ligated GC receptor.

In conclusion, we have shown for the first time that IL-17A induces GC insensitivity in airway epithelium, which is probably mediated by PI3K activation and subsequent reduction of HDAC2 activity. Thus, blockade of IL-17A or downstream signalling molecule PI3K may offer new strategies for therapeutic intervention in GC-insensitive asthma.

KEYWORDS: Airway epithelial cell, airway inflammation, glucocorticosteroids, interleukin-17

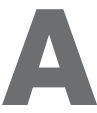
sthma is an obstructive lung disorder, characterised by airway hyperreactivity, airway remodelling and invasion of inflammatory cells, e.g. eosinophils, mast cells and Thelper (Th) cells. Inhaled glucocorticoids (GCs) are currently the most effective anti-inflammatory treatment for asthma. However, a subset of asthmatic subjects is relatively insensitive to this treatment [1,2].

GCs exert a broad spectrum of anti-inflammatory effects upon binding to their receptor (GR). The ligated receptor translocates to the nucleus and suppresses pro-inflammatory gene transcription by recruitment of histone deacetylases (HDACs). HDAC2, in particular, is able to induce deacetylation of histones containing inflammatory genes, thereby restricting access of the transcriptional machinery to these genes and inhibiting transcription [3]. In addition to the recruitment of HDACs, GCs are able to exert anti-inflammatory effects through activation of GC response elements (GREs), which are present in the promoter of several antiinflammatory genes, inducing their transcription.

Reduced sensitivity to GCs has been clinically associated with neutrophilic airway inflammation $[4,5]$, but it is still largely unclear which cellular and molecular mechanisms contribute to this GC insensitivity. Th17 lymphocytes have an emerging role in the induction of neutrophilic airway inflammation [6]. Moreover, Th17-induced neutrophilic airway inflammation in mice was reported to be GC insensitive [7]. Th17 cells act by producing various inflammatory cytokines, including
AFFILIATIONS

*Laboratory of Allergology and Pulmonary Diseases, Dept of Pathology and Medical Biology,

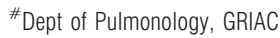
Research Institute, University Medical Center Groningen, University of Groningen, Groningen, The Netherlands.

"These authors share senior authorship.

CORRESPONDENCE

A.J.M. van Oosterhout

Laboratory of Allergology and Pulmonary Diseases Dept of Pathology and Medical Biology

GRIAC Research Institute University Medical Center Groningen University of Groningen P0 Box 30001 9700 RB Groningen The Netherlands

E-mail: a.j.m.van.oosterhout@ med.umcg.nl

Received:

Jan 312011

Accepted after revision:

July 112011

First published online:

Aug 042011 
interleukin (IL)-17A, IL-17F, IL-21 and IL-22, which act locally to induce pro-inflammatory transcription in tissue cells, example IL-8 [6]. Interestingly, IL-17A is known to enhance chemotaxis of neutrophils by inducing IL-8 release in bronchial epithelial cells [8] and airway smooth muscle cells [9]. Despite these novel insights, it is still unknown why GCs are unable to efficiently suppress Th17-mediated inflammation. Although neutrophils are considered relatively unresponsive to GCs, the production of chemokines involved in neutrophilic infiltration, e.g. IL-8 by airway epithelium, can be suppressed by GCs [10].

Therefore, we hypothesise that Th17-mediated GC-insensitive inflammation is not mediated by direct induction of GCinsensitive neutrophil chemoattractant production, but by IL17A inducing epigenetic changes diminishing the possibilities for GCs to inhibit cytokine production. In the present study, we demonstrate that IL-17A significantly reduces GC responsiveness in the human bronchial epithelial cell line $16 \mathrm{HBE}$, which is probably mediated by activation of phosphoinositide-3-kinase (PI3K) signalling and subsequent reduction in HDAC2 activity.

\section{METHODS}

\section{Cell culture}

16HBE14o- human bronchial epithelial cells (16HBE; kindly provided by D.C. Gruenert, University of California, San Francisco, CA, USA) were cultured in flasks coated with $30 \mu \mathrm{g} \cdot \mathrm{mL}^{-1}$ collagen and $10 \mu \mathrm{g} \cdot \mathrm{mL}^{-1}$ bovine serum albumin in Eagle's minimal essential medium (EMEM; Lonza, Walkersville, MD, USA) containing 10\% fetal calf serum (FCS; Hyclone, Logan, UT, USA) and supplemented with $100 \mathrm{U} \cdot \mathrm{mL}^{-1}$ penicillin and $100 \mu \mathrm{g} \cdot \mathrm{mL}^{-1}$ streptomycin (Biowhittaker, Verviers, Belgium), as previously described [11].

\section{Stimulation of the cells}

Cells were seeded in duplicates at a concentration of $10^{5}$ cells $\cdot \mathrm{mL}^{-1}$ in 24 -well plates, grown to $\sim 90 \%$ confluence and serum-deprived overnight. For measurement of IL-8 levels, cells were pre-treated for $2 \mathrm{~h}$ with budesonide (BUD; AstraZeneca, Lund, Sweden) in concentrations ranging from $10^{-11}$ to $10^{-9} \mathrm{M}$, washed and subsequently stimulated with/without $10 \mathrm{ng} \cdot \mathrm{mL}^{-1}$ IL-17A (R\&D Systems, Abingdon, UK) or $10 \mathrm{ng} \cdot \mathrm{mL}^{-1}$ tumour necrosis factor (TNF)- $\alpha$ (Sigma, St. Louis, MO, USA) for $24 \mathrm{~h}$. Alternatively, IL-17A was added $2 \mathrm{~h}$ prior to BUD treatment, in the presence and absence of specific inhibitors of extracellular signal-related kinase (ERK) (U0126, $10 \mu \mathrm{M}$ ), p38 (SB203580, $1 \mu \mathrm{M}$ ) and PI3K (LY294002, $10 \mu \mathrm{M}$ ) pathways, which were added $30 \mathrm{~min}$ prior to IL-17A incubation. All inhibitors were purchased from Tocris Bioscience (Bristol, UK). For measurements of GRE-mediated transcription, cells were incubated for $2 \mathrm{~h}$ with $10 \mathrm{ng} \cdot \mathrm{mL}^{-1} \mathrm{IL}-17 \mathrm{~A}$ and subsequently incubated with BUD in a concentration ranging from $10^{-9}$ to $10^{-7} \mathrm{M}$.

\section{Measurement of IL-8 production}

Cell-free supernatants were harvested $24 \mathrm{~h}$ after stimulation with IL-17A or TNF- $\alpha$. IL-8 production was measured by ELISA (R\&D Systems) according to the manufacturer's instructions.

\section{Immunodetection}

Upon stimulation with/without IL-17A $\left(10 \mathrm{ng} \cdot \mathrm{mL}^{-1}\right)$ for 5 $120 \mathrm{~min}$, total cell lysates were obtained by resuspension of cells in sample buffer ( $2 \%$ sodium dodecyl sulphate (SDS), $10 \%$ glycerol, 2\% 2-mercapto-ethanol, 60 mM Tris- $\mathrm{HCl}$ ( $\mathrm{pH}$ 6.8) and bromophenol blue) and boiling for $5 \mathrm{~min}$. Proteins were separated by SDS-polyacrylamide gel electrophoresis and blotted onto a nitrocellulose membrane. Immunodetection was performed with anti-phospho-p38, anti-phospho-ERK, antiphospho-Akt (Cell Signaling Technology, Hitchin, UK) and pan-ERK (Santa Cruz Biotechnology, Santa Cruz, CA, USA), as previously described [11].

\section{HDAC activity}

Cells were stimulated for $2 \mathrm{~h}$ with $10 \mathrm{ng} \cdot \mathrm{mL}^{-1} \mathrm{IL}-17 \mathrm{~A}$, resuspended in radio-immunoprecipitation assay buffer containing $150 \mathrm{mM} \mathrm{NaCl}, 1 \%$ nonyl phenoxylpolyethoxylethanol$40,0.5 \%$ deoxycholate, $0.1 \%$ SDS and $50 \mathrm{mM}$ Tris $\mathrm{pH} 8.0$, and sonicated to obtain total cell lysates. Protein levels were determined using a BCA kit (Thermo Scientific, Rockford, IL, USA). HDAC activity was assessed by a fluorometric HDAC activity assay (BioVision, Mountainview, CA, USA) performed according to the manufacturer's instructions.

\section{Transfection with GRE-Iuciferase construct}

The GRE-luciferase construct was kindly provided by S.A. Asgeirsdóttir (University Medical Center Groningen, Groningen, the Netherlands). The thymidine kinase-driven renilla luciferase vector (pRL-TK; Clonetech, Paris, France) was used as an internal control. 16HBE cells were grown to $80-90 \%$ confluence in EMEM containing 10\% FCS. For GRE promoter activity measurements, cells were transfected with OptiMEM (Invitrogen, Carlsbad, CA, USA), $1 \mu \mathrm{L} \cdot \mathrm{mL}^{-1}$ Lipofectamine ${ }^{\mathrm{TM}} 2000$ (Invitrogen), $1,000 \mathrm{ng} \cdot \mathrm{mL}^{-1}$ of GRE-luciferase and $100 \mathrm{ng} \cdot \mathrm{mL}^{-1}$ of renilla luciferase construct. After $20 \mathrm{~h}$, cells were washed and stimulated with the indicated amounts of BUD for $24 \mathrm{~h}$. Next, cells were lysed in $50 \mu \mathrm{L}$ passive lysis buffer (Promega, Madison, WI, USA) and subjected to freezing/thawing. Firefly and renilla luciferase activities were determined using the dual-luciferase reporter assay kit (Promega) on a Luminoskan Ascent microplate luminometer (Thermo Scientific) according to manufacturer's instructions. Results were normalised by dividing the firefly luciferase activity with the renilla luciferase activity of the same sample.

\section{Transfection with HDAC2 construct}

Cells were grown to $80-90 \%$ confluence in EMEM containing $10 \%$ FCS, transfected overnight in OptiMEM with $1 \mu \mathrm{L} \cdot \mathrm{mL}^{-1}$ lipofectamine and $1,000 \mathrm{ng} \cdot \mathrm{mL}^{-1}$ of a pcDNA3.1-HDAC2 construct (kindly provided by K. Ito, Imperial College London, London, UK) or an empty vector. After transfection, stimulations were performed as described before.

\section{Statistical analysis}

We tested for normal distribution with the Shapiro-Wilks normality test and observed that TNF- $\alpha$-induced IL- 8 production by $16 \mathrm{HBE}$ cells was normally distributed. We used the t-test for paired observations to test for statistical significance and the ANOVA with Dunnett's post-test when we compared different concentrations.

\section{RESULTS}

\section{IL-17A-induced IL-8 production in 16HBE cells is normally sensitive to BUD}

First, we tested whether IL-17A induced IL-8 production in $16 \mathrm{HBE}$ cells. We observed that treatment with increasing doses 
of IL-17A (24 h) induced an approximate two-fold increase in IL-8 production (fig. 1a). To explore the possibility that IL-17A induced IL-8 production through a GC-insensitive pathway, we tested whether IL-17A-induced IL-8 production was sensitive to BUD. We observed that BUD dose-dependently decreased IL-17A-induced IL-8 production by $16 \mathrm{HBE}$ cells, resulting in a reduction of $49.6 \pm 7.2 \%$ at a concentration of $10^{-9} \mathrm{M}$ (fig. $1 \mathrm{~b}$ ). $10^{-9} \mathrm{M}$ BUD reduced TNF- $\alpha$-induced IL-8 production to a similar extent, e.g. by $46.7 \pm 7.1 \%$ (fig. 2), indicating that IL-17A-induced IL-8 production in bronchial epithelium is normally sensitive to GCs.

\section{IL-17A induces GC insensitivity of TNF- $\alpha$-induced IL-8 production}

Next, we aimed to determine whether IL-17A was able to induce GC insensitivity of TNF- $\alpha$-induced IL- 8 production in $16 \mathrm{HBE}$ cells and we assessed the effect of pre-treatment with $10 \mathrm{ng} \cdot \mathrm{mL}^{-1}$ IL-17A prior to the addition of BUD. $2 \mathrm{~h}$ pre-treatment with IL17A did not affect baseline or TNF- $\alpha$-induced IL-8 levels (fig. 2), but importantly, it significantly reduced the inhibitory effect of $3 \times 10^{-10}$ M BUD from $34.1 \pm 6.7 \%$ to $12.5 \pm 5.8 \%(p=0.006, n=4)$ (fig. 2). Furthermore, we observed that pre-treatment with IL-17 increased the mean inhibitory concentration of BUD on TNF- $\alpha$ induced IL-8 production from $5.20 \times 10^{-10}$ to $1.15 \times 10^{-9} \mathrm{M}(\mathrm{n}=4$, $\mathrm{p}<0.05$; data not shown). This indicates that IL-17A pretreatment reduces sensitivity of TNF- $\alpha$-induced IL- 8 production to BUD. Increasing the period of incubation (up to $6 \mathrm{~h}$ ) or altering the concentration of IL-17A $\left(1-100 \mathrm{ng} \cdot \mathrm{mL}^{-1}\right)$ did not further affect GC sensitivity (data not shown).

\section{IL-17A induces phosphorylation of ERK, p38 and Akt}

To unravel the molecular mechanisms involved in the reduced responsiveness of IL-8 production to BUD upon IL-17A treatment, we first studied whether IL-17A was able to activate PI3K/Akt, mitogen activated protein kinase (MAPK)/ERK kinase (MEK)/ERK-1/2 and p38 MAPK signalling pathways in $16 \mathrm{HBE}$ cells. Immunodetection revealed that IL-17A induced phosphorylation of Akt, ERK-1/2 and p38 in 16HBE cells, with a peak between 5 and $20 \mathrm{~min}$ (fig. 3a). Analysis by densitometry revealed that levels of phosphorylated p-Akt,
p-ERK and p-p38 were significantly increased upon 5 min of incubation with IL-17 (fig. 3b-d).

\section{Inhibition of PI3K abrogates IL-17A-induced GC insensitivity}

Next, we aimed to determine whether activation of these pathways was involved in IL-17A-induced GC insensitivity. We used the specific inhibitors LY294002, U0126 and SB203580 to inhibit the PI3K, MEK/ERK and p38 pathways, respectively. Notably, IL-17A-induced GC insensitivity was, at least partially, reversed upon addition of the PI3K inhibitor LY294002, resulting in stronger inhibition of IL-8 production by BUD in the presence of IL-17A (from $1.8 \pm 9.0 \%$ to $24.9 \pm 6.6 \%, p=0.04$ ). In contrast, the effect of IL-17A on GC sensitivity was not significantly affected by the presence of U1026 and SB203580 (fig. 3e). These data indicate that PI3K, but not ERK and p38, activity contributes to the effect of IL-17A on GC sensitivity.

\section{Reduced HDAC activity may be involved in IL-17A-induced GC insensitivity}

Since PI3K has been implicated in the phosphorylation of HDAC2, leading to its inactivation and a subsequent reduction in GC sensitivity [12], we were interested to see whether IL-17A can reduce HDAC activity in bronchial epithelium. We analysed HDAC activity upon $2 \mathrm{~h}$ of incubation with or without IL-17A. Importantly, IL-17A significantly reduced HDAC activity compared with untreated cells (fig. 4a). In contrast to HDAC activity, IL-17 did not significantly affect total protein expression of HDAC2 (data not shown).

Since HDAC2 has previously been described as the predominant HDAC involved in GC action, we were interested to further underscore the role of HDAC2 in IL-17A-induced GC insensitivity and we studied the effect of IL-17A on GC insensitivity upon overexpression of HDAC2. In cells expressing the control vector, BUD $\left(3 \times 10^{-10} \mathrm{M}\right)$ inhibited TNF- $\alpha$ induced IL- 8 production by $14.2 \pm 4.3 \%$. Pre-treatment with IL-17A significantly reduced this effect to $0.6 \pm 5.4 \%$. In contrast, IL-17A was not able to reduce the effect of BUD in cells overexpressing HDAC2, as BUD still reduced TNF- $\alpha$ induced IL-8 production by $11.1 \pm 3.2 \%$ (fig. $4 \mathrm{~b}$ ). Overexpression
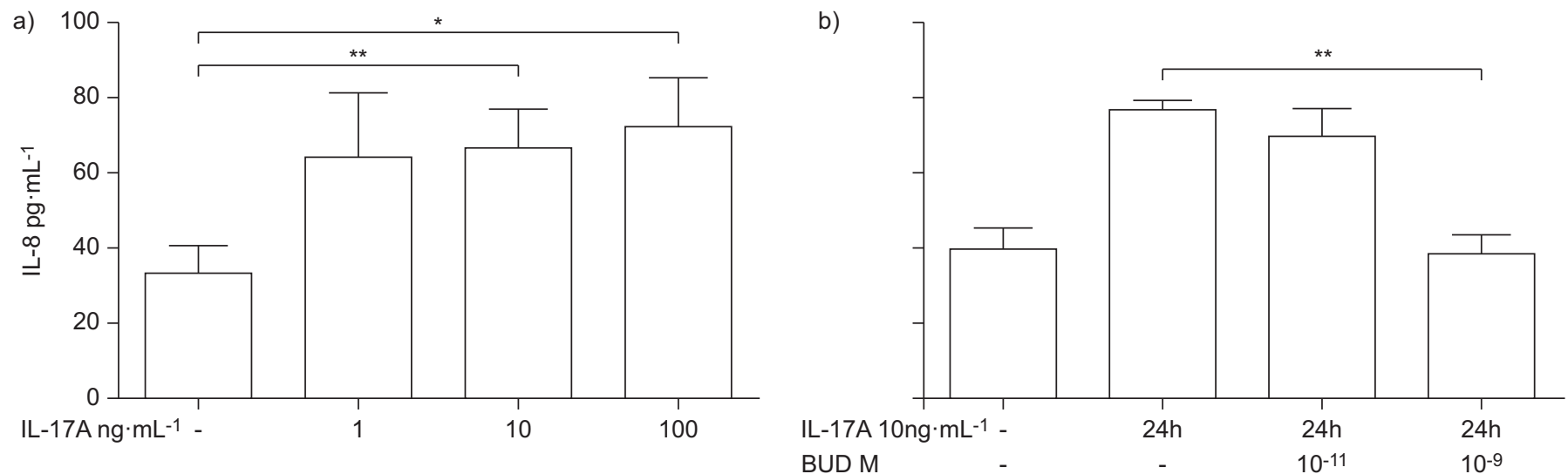

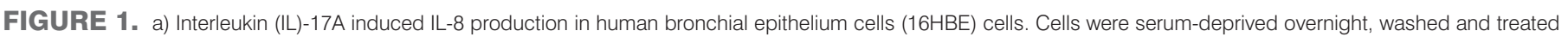

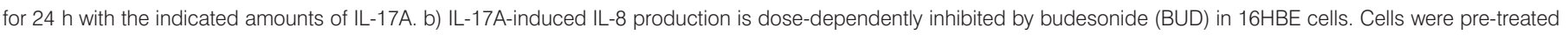

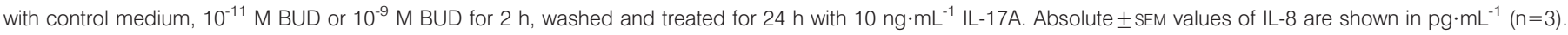
$*: p<0.05$; **: $p<0.01$ between the indicated values. 


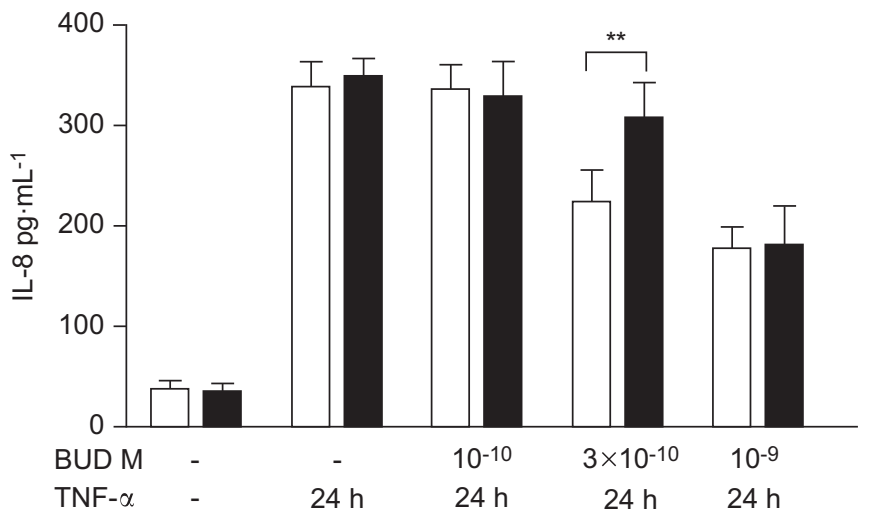

FIGURE 2. Inhibition of tumour necrosis factor (TNF)- $\alpha$-induced interleukin (IL)-8 production by budesonide (BUD) is impaired by pre-incubation with IL-17A. Cells were serum-deprived overnight, pre-treated with $10 \mathrm{ng} \cdot \mathrm{mL}^{-1} \mathrm{IL}-17 \mathrm{~A}(\boldsymbol{\square})$ or control medium $(\square)$ for $2 \mathrm{~h}$, washed, treated for $2 \mathrm{~h}$ with control medium or BUD, washed, and finally incubated for $24 \mathrm{~h}$ with $10 \mathrm{ng} \cdot \mathrm{mL}^{-1} \mathrm{TNF}-\alpha$. Absolute \pm SEM values of IL-8 are shown in $\mathrm{pg} \cdot \mathrm{mL}^{-1}(\mathrm{n}=4)$. ${ }^{*}: \mathrm{p}<0.01$ between the indicated values.

of HDAC1 and HDAC3 did not result in a significant abrogation of the IL-17-mediated effect (see online supplementary fig. 1). These data further support the role for reduced HDAC2 activity in IL-17A-induced GC insensitivity.

\section{IL-17A has no effect on GRE-mediated transcription}

Another mechanism that has been implicated in cytokineinduced GC insensitivity is the reduced translocation of the ligated GR [13]. Nuclear translocation of the ligated GR is required for GCs to exert their suppressive effect on transcription pro-inflammatory genes as well as on transcription of antiinflammatory genes that contain a GRE in their promoter [3, 13]. To test whether IL-17A affects transcriptional activity of the GR, we determined the effect of IL-17A treatment on a GRE reporter construct in 16HBE cells. Our data demonstrated that BUD induced a dose-dependent increase in GRE transcriptional activity, which reached an approximate seven-fold increase at $3 \times 10^{-8} \mathrm{M}$ BUD over basal GRE transactivation $(\mathrm{p}<0.001)$. Pretreatment with IL-17A did not affect BUD-induced activity of the GRE promoter at any of the used concentrations (fig. 5). This indicates that IL-17A does not decrease GRE transcriptional activity or translocation of the ligated receptor within $2 \mathrm{~h}$ of incubation, the time frame in which IL-17A induced GC sensitivity. Therefore, IL-17A-induced GC insensitivity is not likely to be caused by a decrease in translocation of the ligated receptor, but the reduced HDAC activity appears to be the main contributor to this effect.

\section{DISCUSSION}

There is increasing evidence that Th17 cells play a role in the development of GC-insensitive airway inflammation in asthma, although it is still poorly understood why Th17-dependent inflammation is less responsive to GCs. Here, we have shown that IL-17A induced GC insensitivity of TNF- $\alpha$-induced IL-8 production in human bronchial epithelial cells. Furthermore, our data demonstrate that IL-17A-induced IL-8 production is normally sensitive to GCs and that IL-17A does not induce GCinsensitive transcription, as previously suggested [8, 14]. We are the first to show that IL-17A-induced GC insensitivity is mediated by PI3K downstream signalling and a reduction in HDAC activity. As a result, this leads to impaired ability of GCs to efficiently suppress IL-8 production upon stimulation with pro-inflammatory cytokines (e.g. TNF- $\alpha$ ). In contrast to PI3K downstream signalling, the MEK/ERK, p38 MAPK pathways and/or decreased GR translocation to the nucleus do not seem to play a role in IL-17A-induced GC insensitivity in bronchial epithelial cells.

Although inhaled GCs are currently the most effective antiinflammatory treatment of asthma, a subset of patients suffer from a difficult-to-treat and relatively GC-insensitive asthma, which is a burdensome problem in the management of asthma. The mechanisms underlying the poor response in this subset of patients are still largely unknown. Previous studies have shown a link between neutrophilic inflammation and GC insensitivity in asthma [4,5] as well as between neutrophilic inflammation and the presence of Th17 cells in the airways [6]. This led to the hypothesis that Th17-mediated inflammation related to GC-insensitivity. In line with this hypothesis, MCKINLEY et al. [7] showed that the transfer of Th17 cells in mice results in GC-insensitive airway inflammation and airway hyperresponsiveness when compared with the inflammation induced upon the transfer of Th2 cells. Thus, Th17 cells have an emerging role in GC-insensitive neutrophilic inflammation, although the underlying mechanism of GC insensitivity has remained unknown so far.

Our results demonstrate that Th17-induced GC insensitivity is not mediated by activation of GC-insensitive pathways by IL-17A. Similar effects were observed by PRAUSE et al. [15], showing that IL-17A-induced production of IL-8, as well as granulocyte chemotactic protein- 2 and growth-related oncogene- $\alpha$, in 16HBE cells is sensitive to hydrocortisone. In contrast to our findings and those of PRAUSE et al. [15], JONES et al. [8] showed that IL-17Ainduced IL-8 production is insensitive to dexamethasone in primary bronchial epithelial cells. One of the dissimilarities is that JONES et al. [8] seem to have conducted experiments under subconfluent conditions. However, a relationship between cellcell contacts and the actions of GCs has, to our knowledge, not been described. Another important difference is that JONES et al. [8] performed their experiments in primary epithelial cells, while the study by PRAUSE et al. [15] and our study used cell lines. In future studies we plan to compare the effects in cell lines to primary cells, although it may be of concern that the GC hydrocortisone is customarily present in the culture medium for primary cells.

We do not consider it probable that IL-17A plays a major role in the release of IL- 8 in the airways and subsequent development of neutrophilia, since IL-17A was relatively inefficient in inducing IL-8 production when compared with the pro-inflammatory cytokine, TNF- $\alpha$. Thus, we hypothesised that IL-17A exerts a different role in GC sensitivity, reducing the responsiveness of IL-8 transcription to GCs.

In line with this hypothesis, we showed that the pre-incubation with IL-17A reduced the capacity of GCs to inhibit TNF- $\alpha$-induced IL- 8 production. Thus, the presence of IL-17A secreting cells in the airways may render airway epithelial production of IL-8 less responsive to GCs. It is of importance to note that we did not observe an effect on the inhibitory effect of 

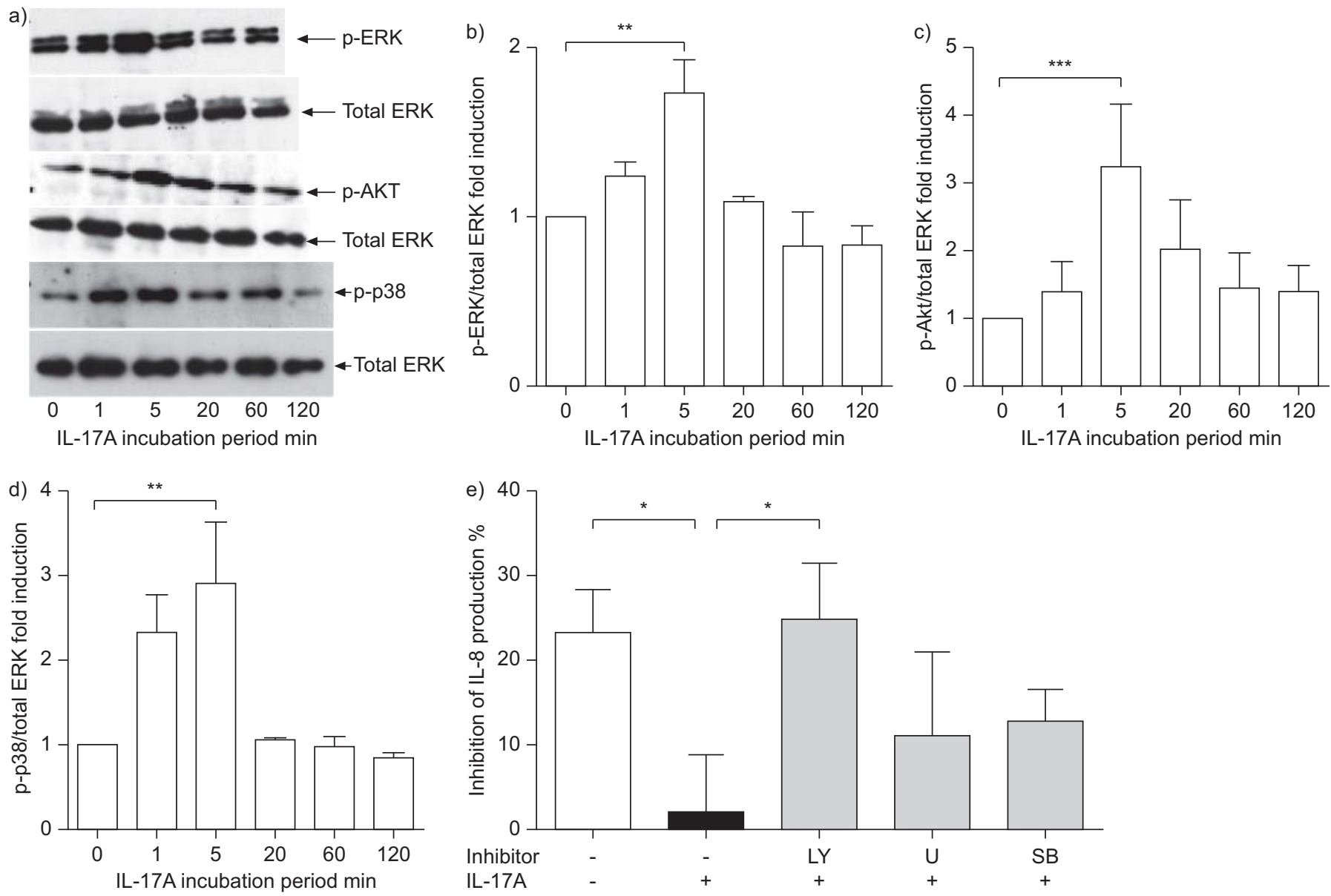

FIGURE 3. a) Interleukin (IL)-17A induced phosphorylation of extracellular signal related kinase (ERK), p38 and Akt. Cells were serum-deprived overnight. IL-17A was added for the indicated period. Phosphorylation (p) of ERK, p38 and Akt was assessed by immunodetection. Representative blots of four independent experiments are shown. Analysis by densitometry of immunodetection of b) phosphorylated ERK ( $p$-ERK), c) phosphorylated Akt ( $p$-Akt) and d) phosphorylated p38 ( $p$-p38) ( $n=4)$. e) An inhibitor of phosphoinositide-3-kinase (PI3K) negates the effects of IL-17A on inhibition of IL-8 production by budesonide (BUD). 30 min prior to IL-17A treatment, the PI3K, ERK and p38 inhibitors were added: LY294002 (LY; $10 \mu \mathrm{M})$, U0126 $(\mathrm{U} ; 10 \mu \mathrm{M})$ and SB203580 (SB; $1 \mu \mathrm{M})$, respectively. $10 \mathrm{ng} \cdot \mathrm{mL}^{-1}$ of IL-17A was added and cells were incubated for $2 \mathrm{~h}$, washed and incubated for $2 \mathrm{~h}$ with $3 \times 10^{-10} \mathrm{M}$ BUD, washed and then incubated for $24 \mathrm{~h}$ with $10 \mathrm{ng} \cdot \mathrm{mL}^{-1}$ tumour necrosis factor (TNF)- $\alpha$. Cell-free supernatants were harvested and IL-8 was determined with ELISA. Data are shown as $\% \pm$ SEM of inhibition by BUD of corresponding TNF- $\alpha$ stimulated control ( $n=4$ ). $*: p<0.05 ; * *: p<0.01 ; * * *: p<0.001$.

BUD at high concentrations. One of the explanations for the lack of effect on the maximum inhibition of BUD is that IL-17A did not affect GR binding to the GRE region. Possibly, the inhibitory effect of GR binding to the IL-8 promoter predominates over the effect of HDAC2 on IL-8 production at higher concentrations of GCs. In addition to our novel findings on the role of IL-17A in GC insensitivity, earlier studies concerning cytokine-induced GC insensitivity have shown possible roles for different cytokines in GC insensitivity, i.e. IL-2 in combination with IL-4 in T-cells [16], IL-13 in monocytes [17], TNF- $\alpha$ in combination with interferon (IFN)- $\gamma$ in airway smooth muscle cells [18] and IL-27 in combination with IFN- $\gamma$ in macrophages [19]. However, none of these cytokines can explain the association between Th17 cells and GC insensitivity, and clinical data on the association between these cytokines and GC insensitivity in asthma is rather scarce. Since IL-17A induces GC insensitivity of IL-8 production in epithelial cells, we postulate that IL-17A itself plays an important role in the development of GC-insensitive neutrophilic inflammation.
We showed that IL-17A-induced PI3K/Akt signalling is involved in the observed effects on GC sensitivity. IL-17A has previously been shown to activate PI3K signalling [20]. Furthermore, PI3K activation has been implicated in $\mathrm{H}_{2} \mathrm{O}_{2}$ - and cigarette smoke-induced GC insensitivity in A549 cells, i.e. by a mechanism involving HDAC2 phosphorylation and subsequent inactivation [12]. In line with this, we found that IL-17A activated the PI3K downstream signalling and decreased overall HDAC activity. Specific overexpression of HDAC2 abrogated IL-17A-induced GC insensitivity, strongly suggesting that PI3K activation and subsequent HDAC2 inactivation play a role in IL-17A-induced GC insensitivity.

In addition to the PI3K-signaling pathway, IL-17A has been described to activate the MEK/ERK signalling in A549 cells and primary tracheal cells $[21,22]$, and both the p38 MAPK and the MEK/ERK signalling in 16HBE cells [23]. We confirmed this in our cells. Activation of the MEK/ERK and p38 pathways has been shown to induce phosphorylation of the GR and decrease 

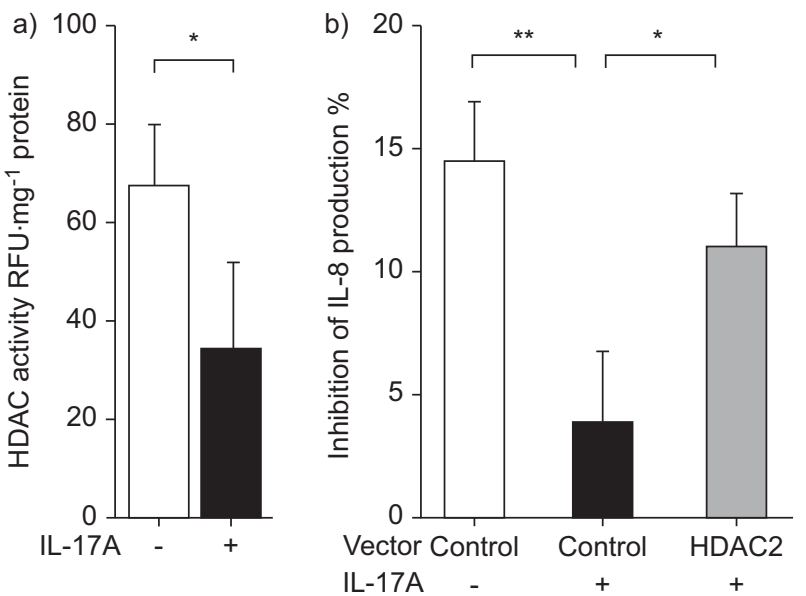

FIGURE 4. a) Interleukin (IL)-17A decreased histone deacetylase (HDAC) activity. Cells were serum-deprived overnight, treated for $2 \mathrm{~h}$ with and without $10 \mathrm{ng} \cdot \mathrm{mL}^{-1} \mathrm{IL}-17 \mathrm{~A}$, resuspended in radio-immunoprecipitation assay buffer and sonicated. HDAC activity was measured using a fluorometric activity assay. HDAC activity is shown as a ratio of the fluorescent units (RFU) per mg of protein $(n=4)$. b) Effect of HDAC2 overexpression on IL-17A-induced glucocorticoid insensitivity. Cells were grown to $90-95 \%$ confluence before transfection with control vector or HDAC2 overexpression. Cells were then pre-treated with $10 \mathrm{ng} \cdot \mathrm{mL}^{-1}$ IL-17A or control medium for $2 \mathrm{~h}$, washed, treated for $2 \mathrm{~h}$ with control medium or budesonide (BUD), washed, and finally incubated for $24 \mathrm{~h}$ with $10 \mathrm{ng} \cdot \mathrm{mL}^{-1}$ tumour necrosis factor (TNF)- $\alpha$. Cell-free supernatants were harvested and IL-8 was determined with ELISA. Data are shown as $\% \pm$ SEM of inhibition by BUD of corresponding TNF- $\alpha$ stimulated control $(n=8) . *$ : $p<0.05 ; * *: p<0.01$.

its translocation to the nucleus causing GC insensitivity in various cell types $[13,24,25]$. However, our data do not support a role for the MEK/ERK and p38 MAPK pathways in IL-17Ainduced GC insensitivity in airway epithelial cells, since specific inhibition of these pathways could not block the IL-17Ainduced effects. Moreover, GRE-mediated transcription was not impaired upon IL-17A exposure in airway epithelium, suggesting that IL-17A-induced MAPK activity does not result in altered translocation of the ligated receptor.

Previous studies in peripheral blood mononuclear cells and broncholaveolar lavage (BAL) fluid of asthmatic patients have suggested that GC insensitivity in asthma patients is associated with increased GR- $\beta[26,27]$, which acts as a negative competitor to the active variant GR- $\alpha$. In line with these findings, VAZQUEZTELLO et al. [14] recently demonstrated that IL-17A induced GR- $\beta$. However, this probably does not contribute to the effect observed in our study, since we observed the strongest effect of IL-17A on GC sensitivity within $2 \mathrm{~h}$ of pre-incubation, whereas VAZQUEZ-TELLO et al. [14] showed that IL-17A does not induce GR- $\beta$ mRNA until after $6 \mathrm{~h}$ in primary bronchial epithelial cells. Moreover, if GR- $\beta$ does play a role in our model, we would expect an effect on the transactivation of the GRE reporter construct due to the competitive nature of this mechanism. Since we did not observe such an effect, we do not render it probable that GR- $\beta$ plays a role in our experiments.

Together with VAzQUEZ-Tello et al. [14], we are the first to describe mechanisms of GC insensitivity in bronchial epithelial cells. Previous studies on GC insensitivity have mainly focused on leukocytes. However, bronchial epithelial cells have an

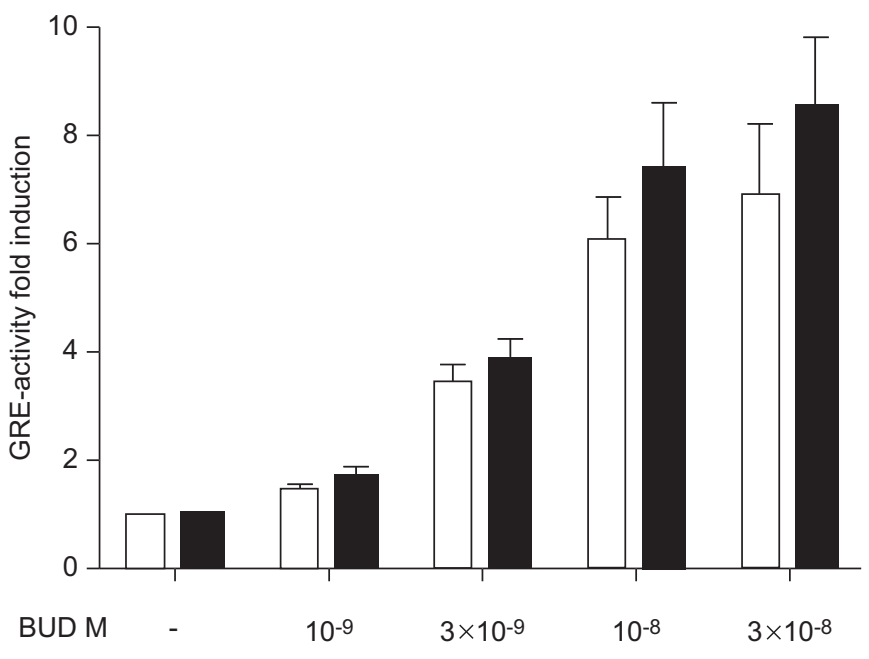

FIGURE 5. Interleukin (IL)-17A has no effect on glucocorticoid response element (GRE)-mediated transcription. Cells were grown to 90-95\% confluence before transfection with GRE-driven firefly luciferase with renilla luciferase as a control for transfection efficiency. Cells were pre-treated for $2 \mathrm{~h}$ with ( $\mathbf{\square})$ or without ( $\square$ ) $10 \mathrm{ng} \cdot \mathrm{mL}^{-1} \mathrm{IL}-17 \mathrm{~A}$, treated for $24 \mathrm{~h}$ with budesonide (BUD) (concentrations $\left.10^{-9}-3 \times 10^{-8} \mathrm{M}\right)$ and resuspended in lysis buffer. Activity was assessed for both luciferases. Data is shown as fold induction \pm SEM over control $(n=4)$.

emerging role in the innate immune response and we speculate that changes in sensitivity to GCs in airway epithelial cells strongly contribute to GC insensitivity of inflammatory responses in the lung.

Cigarette smoking has been associated with a poor response to GCs in asthma [28] and smoking asthmatics displayed increased levels of sputum neutrophils [29]. Interestingly, IL-17A is increased in the BAL of a mouse model of cigarette smokeinduced inflammation [30,31], and therefore it is tempting to speculate that Th17 cells also play a role in smoking-induced GC insensitivity. Strikingly, similar mechanisms have been proposed in smoking-induced GC insensitivity, i.e. PI3K-dependent HDAC2 inactivation [32]. Therefore, it will be of interest to study whether cigarette smoke-induced GC insensitivity is (partly) mediated by IL-17A in future studies.

Given our novel results on the role of PI3K in GC insensitivity, we propose that inhibition of the PI3K pathway may be a promising tool to improve GC function and revert GC insensitivity. In macrophages it has been demonstrated that theophylline treatment restores response to GCs by its inhibitory effect on PI3K activity [33]. Therefore, we anticipate that treatment with theophylline might serve as a strategy to revert GC insensitivity associated with IL-17A, especially given the fact that inhibition of PI3K was also able to restore GC sensitivity in our study. In asthma as well as chronic obstructive pulmonary disease, clinical pilot studies have been performed on the effect of theophylline combined with inhaled GCs [34, 35]. Although these first data show some improvement in lung function, the authors of both studies also stress the need for more extensive investigation into this possible treatment, because both studies were relatively small and single centred. No in vivo and clinical data are available on the action of theophylline on 
Th17-mediated inflammation and the associated steroid insensitivity in severe asthma, thus further studies are warranted.

In conclusion, we have demonstrated that IL-17A is able to induce GC insensitivity in bronchial epithelial cells by activating the PI3K pathway, which may lead to a decrease in HDAC2 activity. Therefore, we propose that therapeutic strategies to inhibit PI3K, as well as therapies focused on downregulating Th17 activity and secretion of IL-17A, may lead to novel ways to improve the efficacy of GCs.

\section{STATEMENT OF INTEREST}

None declared.

\section{REFERENCES}

1 Carmichael J, Paterson IC, Diaz P, et al. Corticosteroid resistance in chronic asthma. Br Med J (Clin Res Ed) 1981; 282: 1419-1422.

2 Schwartz HJ, Lowell FC, Melby JC. Steroid resistance in bronchial asthma. Ann Intern Med 1968; 69: 493-499.

3 Barnes PJ, Adcock IM. Glucocorticoid resistance in inflammatory diseases. Lancet 2009; 373: 1905-1917.

4 Cowan DC, Cowan JO, Palmay R, et al. Effects of steroid therapy on inflammatory cell subtypes in asthma. Thorax 2010; 65: 384-390.

5 Little SA, Chalmers GW, MacLeod KJ, et al. Non-invasive markers of airway inflammation as predictors of oral steroid responsiveness in asthma. Thorax 2000; 55: 232-234.

6 Alcorn JF, Crowe CR, Kolls JK. TH17 cells in asthma and COPD. Annu Rev Physiol 2010; 72: 495-516.

7 McKinley L, Alcorn JF, Peterson A, et al. TH17 cells mediate steroid-resistant airway inflammation and airway hyperresponsiveness in mice. J Immunol 2008; 181: 4089-4097.

8 Jones $\mathrm{CE}$, Chan K. Interleukin-17 stimulates the expression of interleukin- 8 , growth-related oncogene- $\alpha$, and granulocyte-colonystimulating factor by human airway epithelial cells. Am J Respir Cell Mol Biol 2002; 26: 748-753.

9 Vanaudenaerde BM, Wuyts WA, Dupont LJ, et al. Interleukin-17 stimulates release of interleukin-8 by human airway smooth muscle cells in vitro: a potential role for interleukin-17 and airway smooth muscle cells in bronchiolitis obliterans syndrome. J Heart Lung Transplant 2003; 22: 1280-1283.

10 Green RH, Brightling CE, Woltmann G, et al. Analysis of induced sputum in adults with asthma: identification of subgroup with isolated sputum neutrophilia and poor response to inhaled corticosteroids. Thorax 2002; 57: 875-879.

11 Heijink IH, Kies PM, Kauffman HF, et al. Down-regulation of Ecadherin in human bronchial epithelial cells leads to epidermal growth factor receptor-dependent Th2 cell-promoting activity. J Immunol 2007; 178: 7678-7685.

12 Moodie FM, Marwick JA, Anderson CS, et al. Oxidative stress and cigarette smoke alter chromatin remodeling but differentially regulate NF- $\mathrm{KB}$ activation and proinflammatory cytokine release in alveolar epithelial cells. FASEB J 2004; 18: 1897-1899.

13 Irusen E, Matthews JG, Takahashi A, et al. p38 Mitogen-activated protein kinase-induced glucocorticoid receptor phosphorylation reduces its activity: role in steroid-insensitive asthma. J Allergy Clin Immunol 2002; 109: 649-657.

14 Vazquez-Tello A, Semlali A, Chakir J, et al. Induction of glucocorticoid receptor- $\beta$ expression in epithelial cells of asthmatic airways by T-helper type 17 cytokines. Clin Exp Allergy 2010; 40: 1312-1322.

15 Prause O, Laan M, Lotvall J, et al. Pharmacological modulation of interleukin-17-induced GCP-2-, GRO- $\alpha$ - and interleukin-8 release in human bronchial epithelial cells. Eur J Pharmacol 2003; 462: 193-198.
16 Kam JC, Szefler SJ, Surs W, et al. Combination IL-2 and IL-4 reduces glucocorticoid receptor-binding affinity and $\mathrm{T}$ cell response to glucocorticoids. J Immunol 1993; 151: 3460-3466.

17 Spahn JD, Szefler SJ, Surs W, et al. A novel action of IL-13: induction of diminished monocyte glucocorticoid receptor-binding affinity. J Immunol 1996; 157: 2654-2659.

18 Tliba O, Cidlowski JA, Amrani Y. CD38 expression is insensitive to steroid action in cells treated with tumor necrosis factor- $\alpha$ and interferon- $\gamma$ by a mechanism involving the up-regulation of the glucocorticoid receptor $\beta$ isoform. Mol Pharmacol 2006; 69: 588-596.

19 Li JJ, Wang W, Baines KJ, et al. IL-27/IFN- $\gamma$ induce MyD88dependent steroid-resistant airway hyperresponsiveness by inhibiting glucocorticoid signaling in macrophages. J Immunol 2010; 185: 4401-4409.

20 Huang F, Kao CY, Wachi S, et al. Requirement for both JAKmediated PI3K signaling and ACT1/TRAF6/TAK1-dependent NF- $\kappa B$ activation by IL-17A in enhancing cytokine expression in human airway epithelial cells. J Immunol 2007; 179: 6504-6513.

21 Ning W, Choi AM, Li C. Carbon monoxide inhibits IL-17-induced IL-6 production through the MAPK pathway in human pulmonary epithelial cells. Am J Physiol Lung Cell Mol Physiol 2005; 289: L268-L273.

22 Inoue D, Numasaki M, Watanabe M, et al. IL-17A promotes the growth of airway epithelial cells through ERK-dependent signaling pathway. Biochem Biophys Res Commun 2006; 347: 852-858.

23 Laan M, Lotvall J, Chung KF, et al. IL-17-induced cytokine release in human bronchial epithelial cells in vitro: role of mitogenactivated protein (MAP) kinases. Br J Pharmacol 2001; 133: 200-206.

24 Tsitoura DC, Rothman PB. Enhancement of MEK/ERK signaling promotes glucocorticoid resistance in CD4+ T cells. J Clin Invest 2004; 113: 619-627.

25 Onda K, Nagashima M, Kawakubo Y, et al. Mitogen-activated protein kinase kinase 1/extracellular signal-regulated kinase (MEK-1/ERK) inhibitors sensitize reduced glucocorticoid response mediated by TNF- $\alpha$ in human epidermal keratinocytes (HaCaT). Biochem Biophys Res Commun 2006; 351: 266-272.

26 Leung DY, Hamid Q, Vottero A, et al. Association of glucocorticoid insensitivity with increased expression of glucocorticoid receptor ß. J Exp Med 1997; 186: 1567-1574.

27 Goleva E, Li LB, Eves PT, et al. Increased glucocorticoid receptor $\beta$ alters steroid response in glucocorticoid-insensitive asthma. Am J Respir Crit Care Med 2006; 173: 607-616.

28 Thomson NC, Chaudhuri R, Livingston E. Asthma and cigarette smoking. Eur Respir J 2004; 24: 822-833.

29 Chalmers GW, MacLeod KJ, Thomson L, et al. Smoking and airway inflammation in patients with mild asthma. Chest 2001; 120: 1917-922.

30 van der Deen $M$, Timens W, Timmer-Bosscha H, et al. Reduced inflammatory response in cigarette smoke exposed Mrp1/Mdr1a/ 1b deficient mice. Respir Res 2007; 8: 49.

31 Melgert BN, Timens W, Kerstjens HA, et al. Effects of 4 months of smoking in mice with ovalbumin-induced airway inflammation. Clin Exp Allergy 2007; 37: 1798-1808.

32 Marwick JA, Caramori G, Casolari P, et al. A role for phosphoinositol 3-kinase $\delta$ in the impairment of glucocorticoid responsiveness in patients with chronic obstructive pulmonary disease. J Allergy Clin Immunol 2010; 125: 1146-1153.

33 To Y, Ito K, Kizawa Y, et al. Targeting phosphoinositide-3-kinase- $\delta$ with theophylline reverses corticosteroid insensitivity in chronic obstructive pulmonary disease. Am J Respir Crit Care Med 2010; 182: 897-904

34 Spears M, Donnelly I, Jolly L, et al. Effect of low-dose theophylline plus beclometasone on lung function in smokers with asthma: a pilot study. Eur Respir J 2009; 33: 1010-1017.

35 Ford PA, Durham AL, Russell RE, et al. Treatment effects of lowdose theophylline combined with an inhaled corticosteroid in COPD. Chest 2010; 137: 1338-1344. 